\title{
Version Control in Open Source Software
}

\author{
Gurpal Singh( Mtech CSE) \\ SSCET Badhani (pathankot)
}

\begin{abstract}
The Open source software can be redistributed to others users and they can use it according to their own needs. Version Control is the process which is most commonly development and with the help of this process, a team of people may change the same files at the same time or at different time. Each Version is associated with a time stamp and the and persons making the change. There are many open source software like open Office, Mozilla Fire Fox etc. But inthis dissertion, Jfreechart have been taken and the variation of classes in each version are observed. Various Metrics have been Observed with the help of CCC tool such as Line of Comments, coupling, number Of Children and the depth of inheritance tree
\end{abstract}

Keywords: Basic Introduction, examples of open Source software's, open source software licenses, Advantages Using Open Source Software, General Idea of Version Control, JFree Chart, Various Versions In J Free Chart

\section{Basic Introduction}

1. Open Source Software are those software whose source code is freely available( Without any Interrupt of payment Or any other obstacles)

2. The Open Source Software is a software that must be distributed with source code included or easily available such as by free down load from internet

3. The State Of Kansas Published a definition : OSS is software for which the source code is freely and publicly available ,though the specific licensing agreement vary as to what one is allowed to do with that code

The main features that characterize open source Software is the freedom that user have to:

- Use the software as they wish, for whatever they wish, on as many computers as they Wish, in any technically appropriate situation

- Have The Software at their disposal to fit it to their needs .Of Course, this include improving it, fixing its bugs ,augmenting its functionality studying its operation

- Redistribute the software to other users, who could themselves use it according to their own needs. This redistribution can be done for free, or at a charge, not fixed beforehand

\section{Examples Of Open Source Software:}

1. Open Office.org_ Office Suite

2. Mozilla And Fire Fox- Web Browser

3. Thunder Bird- email-client

4. Tex/Latex-Typesetting and Document preparation System

5. My Sql- Relational Database Systems

6. GNU/LINUX-Operating Systems

7. Apache Web Server- Server

\section{Open Source Software Licences:}

Licenses usually specify that the proprietor of the program is the company which publishes it, which just sells restricted rights to use it .Usually, the conditions specified in licenses of open source software are the result of a compromise between several goals which are in some sense contradictory .Among them the following can be cited

- Guarantee some basic freedoms (redistribution, modification, use ) to the users

- Ensure Some Conditions imposed by the authors

- Guarantee that derived Works are also open source software 


\section{Some Common Open Source Software Licenses Are: 4.1BSD (Berkley Software Distribution): \\ BSD license is good example of a license, which imposes almost no conditions on what a user can do with the software, including charging clients with no obligations to include source code ,in other words redistributors can do almost anything with the software, including using it for propriety products .}

\subsection{GPL (General Public License):}

This is the license under which the software of the GNU project is distributed. The GPL was carefully designed to promote the production of more free software, and because of that it explicitly forbids some actions on the software which could lead to the integration of GPLED software is the propriety programs .The GPL is based on the international legislation on copy right, which ensures its enforceability

\subsection{MPL (Mozilla Public License):}

This is the license made by Netscape to distribute the code of Mozilla , the new version of it network navigator .It is in many respects similar to the GPL, but perhaps more enterprise

Oriented

Other Well Known Licenses are the (written by Troll Tech, the Authors of the Qt Library),

The artistic license and the X Consortium license

\section{Advantages Of Open Source Software:}

5.1 The availability of the source code and the right to modify it: It Enables the unlimited tunneling and improvement of a software product .It also makes it possible to port the code to new hardware,to adapt it to changing conditions , and to reach detailed understanding of how the system works. This is why many researchers concluded that to really extend lifetime of an application.

5.2 The right to redistribute modifications and improvement to the code : To reuse other Open Code permits all the advantages due to modification of the product should be shared by large .This is usually the point that differentiates open source software licenses from nearly free one .In Substance, the fact that redistribution rights cannot be revoked

\section{The Right of Software in any way:}

This Combined with redistribution rights, ensures a large population of users, which helps in turn to build up market for support and customization, of the software which can only attract more developers to work in the project

\section{Version Control In Open Source Software:}

A version control system is combination of technologies and practices for tracking and controlling changes to a project's file, in particular to source code, documentations and web pages . These days, everyone will expect at least the project source code to be under version control .

The reason of version control is universal is that helps with virtually ever aspect of running a project : inter developer communications, release management, bug management, code stability ,and experimental development efforts, and authorization of changes .The Version control System provides a central coordinating force among all these areas

\subsection{Need of version Control:}

The need for the software version control rises .As the software is developed and deployed it is extremely common for multiple versions of the same software to be deployed in different sites and for software developers for working privately on updates .Bugs and other issues in software are often only present in certain versions. Therefore for purpose of Locating Bugs, it is vitally important for debugger to be able to retrieve and run different versions of the software concurrently, where one versions has bugs fixed, but no new features, while the other versions are is where new features are worked on .

\subsection{Advantages of version Control:}

Version Controls many advantages to both team and individuals as follows:

- It gives team project wide Undo Buttons i.e. nothing is final and mistakes can be easily rolled back

- It allow developer to work It on same code base in a controlled manner

- It keeps record over changes made over a period of time .It is easy to find out changes when and why

- It Supports multiple releases of software at the same time 
- It acts like a project wide time machine, allows us to dial in a date and see exactly what the project look like on that date

\section{JFree Chart :}

The open source software that has been used in this dissertation is Jfree chart

JFree chart is a free $100 \%$ java chart library that makes it easy for developer to display professional quality charts in their application .JFree chart extensive features set includes:

- Consistent and well documented API, supporting a wide range of chart types

- Flexible design that is easy to extend and targets both server sides and client side applications

- Supports for many output types, including swing components, image files( including PNG and JPEG ) and vector graphic Formats( including PDF ,EPS, and SVG

- Jfree chart is open source or more specifically, free software . It is distributed under the terms of GNU Lesser General Public License (LGPL), which permits use in the propriety applications

Various Versions Of Jfree Chart:

1. Jfree chart 0.019

2. Jfree chart 0.9 .21

3. Jfree chart 1.0.1

4. Jfree chart 1.0.2

5. Jfree chart 1.0 .3

6. Jfree chart 1.0.4

7. Jfree chart 1.0 .5

8. Jfree chart 1.0.6

9. Jfree chart 1.0.7

10. Jfree chart 1.0 .8

11. Jfree chart 1.0 .9

12. Jfree chart 1.0 .10

13. Jfree chart 1.011

14. Jfree chart 1.0.12

15. Jfree chart 1.013

The source code of each version has been studied and there are different number of packages used on each version . three common packages has been taken into consideration and the number of classes have been compared with each other using different metrics .

Different Packages in each version :

\begin{tabular}{llll}
\hline \multicolumn{1}{c}{ Version 0.9.19 } & \multicolumn{1}{c}{ Version 0.9.21 } & Version 1.0.1 & Version 1.0.2 \\
\hline $\begin{array}{l}\text { 1.Category Annotation } \\
\text { Clame number of } \\
\text { 2.Category Text Annotation } \\
\text { With Respect to the } \\
\text { Previous Veion }\end{array}$ & $\begin{array}{l}\text { Four classes are } \\
\text { Added the previous } \\
\text { Version These Are } \\
\text { As Follows: }\end{array}$ & $\begin{array}{l}\text { Same number of class } \\
\text { As compared to other versions }\end{array}$ \\
$\begin{array}{l}\text { 1.XY Box Annotation } \\
\text { 4.XY Annotation }\end{array}$ & 2.Abstract XY Annotation \\
5.XY Draw able Annotation & 3.XY Polygon Annotation & \\
6.XY Image Annotation & 4.Category Line Annotation
\end{tabular}

7.XY Line Annotation

8.XY Pointer Annotation

9.XY Shape Annotation

10.XY Text Annotation 
Table 2.

\begin{tabular}{|l|l|l|l|}
\hline $\begin{array}{l}\text { Version 1.0.3 } \begin{array}{l}\text { Class has been added W.R.T } \\
\text { the previous versions }\end{array} \\
\text { Category pointer Annotation }\end{array}$ & $\begin{array}{l}\text { Version1.0.4to1.0.10 } \\
\text { Same number of classes has } \\
\text { been used as compared to } \\
\text { previous versions }\end{array}$ & $\begin{array}{l}\text { Two classes have been } \\
\text { added } \\
\text { XY Data Image } \\
\text { Annotation } \\
\text { XY Title Annotation }\end{array}$ & $\begin{array}{l}\text { 1.class has been added } \\
\text { XY Annotation Bounds Info }\end{array}$ \\
\hline
\end{tabular}

Table 3.

AXIS

\begin{tabular}{|l|l|l|l|}
\hline Version 0.019 & Version 0.9.21 & Version 1.0.1 & Version 1.0.2 \\
\hline \multicolumn{1}{|c|}{ Axis Space } & $\begin{array}{l}\text { 3 More classes have been } \\
\text { added in this version as } \\
\text { compared to other Versions }\end{array}$ & $\begin{array}{l}\text { 2. Classes have Been } \\
\text { added and one class has been } \\
\text { removed The Removed Class } \\
\text { is }\end{array}$ & $\begin{array}{l}\text { Same number of classes } \\
\text { have been used as compared } \\
\text { to previous Versions } \\
\text { 2.category Axis 3D }\end{array}$ \\
$\begin{array}{l}\text { 3Category Tick } \\
\text { 4.Date Axis }\end{array}$ & Period axis \\
5.Extended Category Axis & Modulo axis & $\begin{array}{l}\text { And the added Classes are : } \\
\text { Month Date Format }\end{array}$ & Quarter Date Format \\
6.Number Axis 3D & & \\
7.Segemented Time Line & & \\
\hline
\end{tabular}

\section{Conclusion:}

Version Control is veryimportant activity to access and monitor the evolution of aparticular product.It Becomes very essential to keep track of various versions over a period of time .Different Versions of JFree Chart have been studied and based upon different metrics, it is concluded that as the lines of code increases, the cyclomatic complexity also increases. Therefore, of LoC is increased accumulatively from the previous versions to the next version i.e if there is a sudden rise in the value of LOC that means they have not been significant additions to the product. Similarly, weighted methods per class is the sum of McCabe's number . Therefore, it remains constant with the Mc Cabe's complexity and coupling between objects almost remains constant during each packge which is significant.

\section{Future Directions:}

As different Versions of a open source software ,J Free Chart has been analyzed .15 versions of this software have been taken various metrics have calculated. But if better results are required and if results are required on a broad basis, then larger number of versions can be taken. The bigger number of versions is better will the results

\section{References}

[1]. M.Pilato "Version Control With Sub Version, o.reilly and associates

[2]. The origin and future of Open Source Software by net action white paper by Nathan New man

[3]. Joseph Jeller ,Brain Fitz Gerald “ Understanding Open Source Development 2002 P.P15-150 ISBN

[4]. Ben Collins .Sussman, Brain W. Fitzpatrick,C. Michael Pilato

[5]. Joseph Jeller, Brain Fitzgerald $2006>$ White Paper on Version Control

Some Important Referred Web sites:

1. www.linfo.org

2. Www.gnu.org

3. Www.opensource.org

4. Www.jfree.org

5. www.ccc.sourceforge.net

6. Www.stders.org

7. www.eu.conecta.it/paperAdantages open -source software 